\title{
SYNTHESIS AND OPTIMIZATION OF SUBMARINE PIPELINE ROUTES
}

\author{
J. S. Baioco ${ }^{1}$, D. C. S. Coutinho ${ }^{1}$, C. H. Albrecht ${ }^{1}$, B. S. L. P. de Lima ${ }^{1}$, B. P. Jacob ${ }^{1}$, D. M. \\ Rocha $^{2}$, C. O. Cardoso ${ }^{3}$
}

${ }^{1}$ LAMCSO - Laboratory of Computer Methods and Offshore Systems, COPPE/UFRJ - Post Graduate Institute of the Federal University of Rio de Janeiro, Civil Engineering Dept.

${ }^{2}$ PETROBRAS Research and Development Center (djalene@petrobras.com.br)

\section{${ }^{3}$ PETROBRAS Research and Development Center}

\begin{abstract}
Traditionally, the selection of a pipeline route for offshore applications has been manually performed by the engineer, by a quick inspection of the seabottom bathymetry and the available information regarding obstacles. Eventually the evaluation of a given route could be performed using analysis tools, but in any case the process is highly dependent on the expertise of the engineer. In this context, this work describes the development of a computational tool for the synthesis and optimization of submarine pipeline routes, using computational tools based in Evolutionary Algorithms.

In such optimization procedures, each candidate route is randomly generated and is evaluated, in order to determine its "fitness", in terms of several criteria that are incorporated in an objective function. Such function takes into account all relevant aspects that should be considered in the selection of a route, such as total pipeline length; geophysical and geotechnical data obtained from the bathymetry and sonography, including the definition of obstacles and regions that should be avoided; number, length and location of free spans to be mitigated along the routes. Other aspects depend on the structural behavior of the pipe, under hydrostatic and environmental loadings; some of these aspects are dealt with by following recommendations established in the DNV RP-F105 and RP-F109 codes, related respectively to the on-bottom stability and free spans.

This work describes the implementation of the optimization tool, beginning with the assembly of the objective function and the definition of the problem constraints, and proceeding with the association of this function and constraints in the framework of the implementation of a Genetic Algorithm - GA. Case studies are presented to illustrate the use of this optimization tool. It is expected that the application of such tool may reduce the design time needed to assess an optimal pipeline route, while reducing computational overheads and providing more accurate results (avoiding mistakes with route interpretation), ultimately minimizing costs with respect to submarine pipeline design and installation.
\end{abstract}

Keywords: Pipeline Routes; Optimization, Evolutionary Algorithms. 


\section{INTRODUCTION}

Traditionally, the selection of a pipeline route for offshore applications has been manually performed by the engineer, by an inspection of the seabottom bathymetry and the available information regarding obstacles. Eventually the evaluation of a given route could be performed using analysis tools, but in any case the process is highly dependent on the expertise of the engineer. Therefore, it should be recognized that the selection a submarine pipeline route with good performance and low cost must indeed be formally described and treated as a synthesis and optimization problem.

Intuitively, the best pipeline route for offshore applications would be the one with smaller length, leading to lower material costs. However, other factors that affect the performance of the route must also be considered, including for instance geophysical / geotechnical data obtained from the seabed bathymetry and sonography. These data defines the obstacles and regions that should be avoided; leads to a minimum radius of curvature for the route, and also to the number of free spans along the route that should be minimized/mitigated.

Thus, it can be seen that the selection of a submarine pipeline with high performance and low cost should indeed be formally described and treated as a synthesis and optimization problem. Optimization methods seek to find an optimal solution to a given engineering problem within a set of solutions, usually subject to constraints. Nature-inspired algorithms such as the Genetic Algorithms (GA) have been shown to be very useful for the search and optimization of solutions for offshore engineering problems [1],[2],[3].

In this context, this work describes the evolution of a computational tool for the synthesis and optimization of submarine pipeline routes. A previous work by the authors [4] described the initial steps taken towards the development and implementation of a computational tool. This work [4] was focused on the geometrical representation of a route, and on some of the terms of the objective function associated with a preliminary stage of the optimization process (such as total pipeline length, and geographical-topographical issues associated with the route geometry and to the seabottom bathymetry and obstacles).

A second work [5] focused on other aspects related to the structural behavior of the pipe, under hydrostatic and environmental loadings. More specifically, special attention is dedicated to the implementation of On-Bottom Stability (OBS) criteria such as the proposed in the DNV-RP-F109 code [6].

A third work [7] aimed at analyzing Absolute Lateral Static Stability verifies the required ballast weight for achieving stability hydro-dynamics in order to optimize the minimum ballast layer simultaneously with the route length.

This work is focused in a review of the evolution of a computational tool. Initially, the next section describes the optimization method selected (Genetic Algorithms - GA). After that, presents the parameterization considered for the geometric representation of the configuration of a pipeline route. Next, follows the description of the components of the objective or fitness function (that evaluates each candidate route randomly generated during the search process) and the problem constraints and penalties. Next, presents the On-Bottom Stability Criterion used in the studies. Lastly, case studies are presented to illustrate the use of this optimization tool. 


\section{OPTIMIZATION METHOD}

As mentioned before, the optimization method applied at the current phase of the research is the Genetic Algorithm (GA), which is a mathematical model inspired by the evolution of species [8]. In this methodology, randomly generated candidate routes are individuals of a population, with each individual being represented as a chromosome. Each chromosome encodes a candidate solution via its genes, each corresponding to one of the parameters that define a route.

To verify the quality of an individual, it is evaluated according to its efficiency (or fitness). The evaluation is performed by means of an objective function, taking into account the constraints of the problem, as will be described later in this work.

For the generation of each new population, the individuals are ranked according to their fitness; only the fittest individuals of the current population are selected to continue in the evolution process, and the others are discarded. Selected individuals are subjected to the genetic operators for the reproduction process, by crossing parents of the current population, which exchange genes to produce offsprings. Subsequently, the offsprings may be subjected to the mutation process, which confers different characteristics to the individual and provide a better exploration of the problem search space.

This process ends when a pre-defined stopping criterion is reached, and the individual with the best fitness is then defined as the solution of the problem. The algorithm is summarized schematically in Fig. 1.

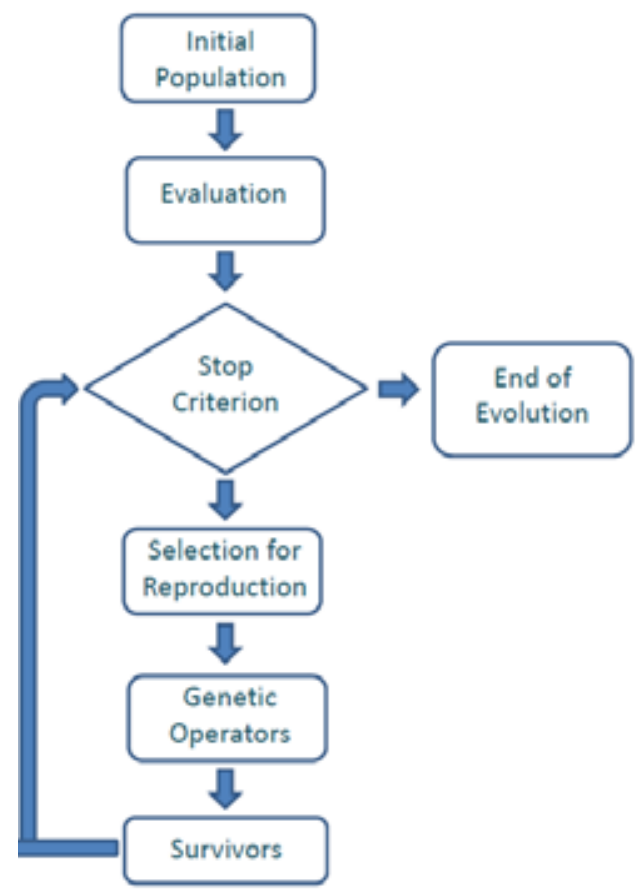

Figure 1. Genetic Algorithm Flowchart. 


\section{PROBLEM DESCRIPTION}

\subsection{Geometric Representation of Submarine Pipeline Route}

This section summarizes the parameterization method employed for the geometric representation of a given pipeline route configuration. Considering that the endpoints of a given route are represented by points $\mathrm{A}$ and $\mathrm{B}$, ideally the straight line $\mathrm{AB}$ connecting these points would be the shortest and best route. However, actual routes must incorporate curved sections in order to bypass obstacles crossing this line, or to comply with other constraints or criteria that will be defined later.

In the geometric representation defined for the optimization tool, the curves are defined as circular arcs, as illustrated in Fig. 2. To each curve is associated an Inflexion Point (PI), that is represented by:

a) Curvature radius $R$, and

b) Coordinates Radial $(\delta)$ and Angular $(\alpha)$ of the corresponding inflexion point PI (Fig. 3).

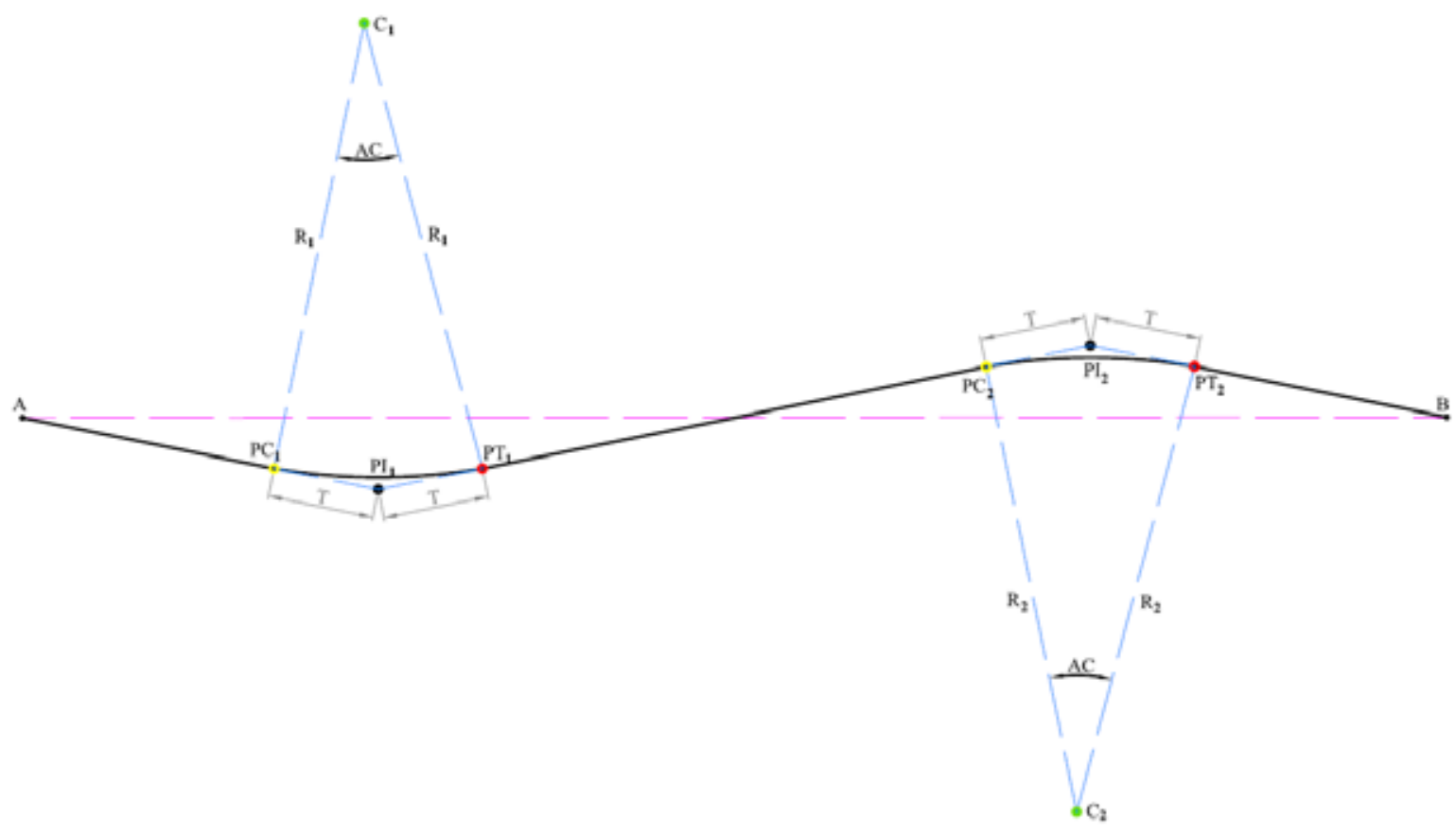

Figure 2. Geometric representation.

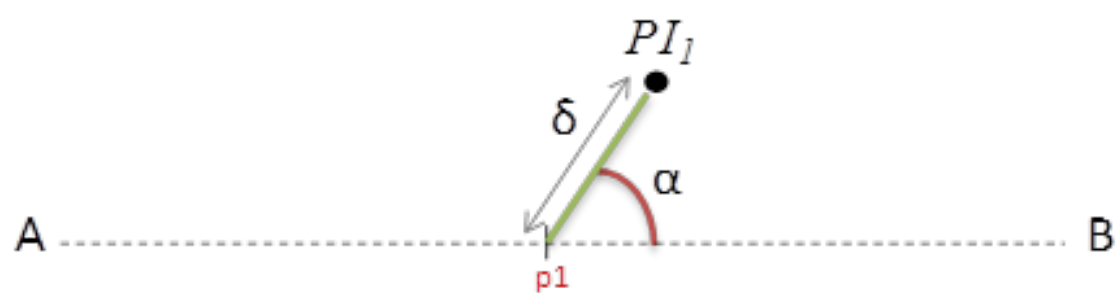

Figure 3. Polar Coordinates defining a PI. 
The Genetic Algorithm codification of each individual is then comprised by a chromosome with $\mathrm{N}$ sets of genes, each set associated with a PI, with three genes describing the main parameters described above (the coordinates $\delta$ and $\alpha$, and the curvature radius $\mathrm{R}$ ).

Moreover, there is a fourth gene, associated with an activation key A that indicates the status of each PI. This allows the number of PIs to vary along the optimization process, beginning with a maximum number specified by the user. Therefore, depending on the complexity of the problem, the geometric representation of the route can be simplified by the algorithm, by disabling some PIs, that is, considering that the corresponding section of the route is straight. The activation key A is a binary value 0 (indicating that the PI is inactive and its parameters should be ignored) or 1 (indicating that the PI generates a curve). The full codification of a chromosome can then be written as:

$$
A_{1} \delta_{1} \alpha_{1} R_{1} A_{2} \delta_{2} \alpha_{2} R_{2} \ldots A_{N} \delta_{N} \alpha_{N} R_{N}
$$

where $\mathrm{A}_{1} \delta_{1} \alpha_{1} \mathrm{R}_{1}$ are the genes corresponding to the first PI, and $\mathrm{N}$ is the maximum number of PIs specified by the user. Therefore, along the process of generating an optimal route, the algorithm will define candidate solutions by selecting the number of points PI, the values for the coordinates that define their position, and the associated curvature radius.

\subsection{Objective Function}

Having described the codification of each candidate solution in the optimization process, it is necessary to define an objective function to evaluate the suitability of each candidate route. The objective function should reflect the fitness or "quality" of the route, taking into account the relevant aspects concerning the choice of the best route.

\subsubsection{Variable Optimized: Route Length}

One of the more important factors involved in the evaluation of a route is its total length. The length of the pipeline should be minimized, in order to reduce material costs; if other aspects were ignored, the best route would be trivially defined by the straight line connecting points A and B. Of course there are several other factors that influence the cost and safety of a pipeline route, including physical, geometric and structural constraints, related for instance to geographical/topographical issues associated with the seabottom bathymetry, interference with obstacles, slope and so on.

These and other factors are treated by introducing penalty terms to the objective function, whenever a given constraint is violated. Therefore, the objective or fitness is calculated by the following expression:

$$
\text { fitness }=100 \cdot \frac{\text { dist }_{A B}}{L_{\text {Route }}+\text { dist }_{A B} \sum_{i=1}^{N p}\left(k_{i} \cdot \text { penal }_{i}\right)}
$$

Since the goal of the optimization process is the minimization of the route length $L_{\text {Route }}$, subjected to $N p$ penalty terms, this expression represents a maximization function. The term dist $_{A B}$ represents the length of the straight line between the end points of the route. If a given route does not violate any constraint, its fitness is defined as dist ${ }_{A B} / L_{\text {Route }}$, and the trivial solu- 
tion of a non-penalized straight route would therefore has the maximum fitness value of 1 since $\operatorname{dist}_{A B}=L_{\text {Route }}$. The constant 100 is introduced as a scale factor, in order to define the range of possible fitness values as $[0,100]$.

The value of each penalty term i $[1, N p]$ is represented as penal ${ }_{i}$, and $k_{i}$ represents a weight factor that can be assigned to each penalty in order to control the relative influence of each constraint.

\subsubsection{Variable Optimized: Route Length and Ballast Weight}

To deal with the problem of the minimum required ballast weight to keep the pipe hydrodynamically stable and to minimize route length, a fitness formulation that includes both ballast weight and pipe length portions was designed:

$$
\begin{aligned}
\text { fitness } & =100 \cdot \frac{k_{\text {pipe }} \text { dist }_{A B}}{k_{\text {pipe }} L_{\text {Route }}+k_{\text {ballast }} W_{\text {reqTotal }}+\text { dist }_{A B} \sum_{i=1}^{N p}\left(k_{i} \cdot \text { penal }_{i}\right)} \\
W_{\text {reqTotal }} & =\sum_{i=1}^{n E l e m} W_{\text {req }}(i) \cdot L_{\text {elem }}(i)
\end{aligned}
$$

where fitness is the route evaluation function; dist $_{A B}$ is the $\overline{A B}$ line length; $L_{\text {Route }}$ is the total length of the route; $k_{\text {pipe }}$ is the pipe length weighting factor; $k_{\text {ballast }}$ is the ballast weighting factor; $W_{\text {reqTotal }}$ is the total ballast weight required for the route; $n E l e m$ is the number of elements that compose the route; $W_{\text {req }}$ is the ballast weight required to stabilize a route element; $L_{\text {elem }}$ is the length of a route element; $N p$ is the number of penalties; penal ${ }_{i}$ is the value of each $i$ penalty $[1 ; N p] ; k_{i}$ is the weighting factor applied to each penalty, biasing the role of each penalty in the final result.

By means of this formulation it is possible to concurrently optimize both the route length and the minimum required ballast weight, the weighting factors being the designer's way to prioritize one over another. That is, if the ballast weight is the most important factor in a project due to difficulty in providing ballast in a certain region in which the pipe should be laid, the designer may increase $k_{\text {ballast }}$ in order to ensure a stable route with a lower ballast weight . In case the limitation is steel and the route length is the predominant factor, $k_{\text {pipe }}$ may be increased to decrease route length.

\subsection{Constraints}

The current implementation of the optimization tool considers penalty terms associated with geographical-topographical aspects related to the geometry of the route, to the seabottom bathymetry and to the interference with obstacles.

\subsubsection{Self-Crossing}

With the parameterization described before, the optimization process might eventually generate routes with a geometric representation in which the pipeline passes over itself. A 
penalty term is then introduced to avoid configurations with self-crossings or loops, by employing an algorithm that spans all segments of the route and counts the number of selfcrossings $n$ SelfCross.

$$
\text { penal }_{\text {SelfCross }}=\exp (\text { nSelfCross })-1
$$

\subsubsection{Interference with Obstacles}

The seafloor regions around the routes may include critical areas such as regions with corals, geotechnical hazards, or other obstacles such as subsea equipments and flowlines. It should also be considered that, while interference with some of these obstacles may be tolerable, some other obstacles cannot be crossed at all.

In the current implementation of the tool, four types of obstacles are being considered:

- Line (e.g. another pipeline) - defined by segments connecting points with coordinates $(\mathrm{x}, \mathrm{y}, \mathrm{z})$;

- Polygon - representing a closed region, corresponding for instance to a geohazard, and defined by vertices with coordinates $(\mathrm{x}, \mathrm{y}, \mathrm{z})$ connected by segments;

- Point - in fact a spherical volume defined by its center with coordinates (x,y,z) and a radius $r$; and

- Cylinder - a volume defined by its center with coordinates (x,y,z), a radius $r$ and a height $z$, oriented in the vertical direction.

During the optimization process, interference is identified by verifying the intersections between the segments of each generated route against the segments or volumes that define the obstacles. The total number of intersections is counted and assigned to a variable nInter. Routes that cross obstacles are then penalized, considering also different levels of importance that may be attributed to each obstacle, according to the consequences of the possible interference (Fig. 4):

- Level 0 (gray) - Used for illustrative obstacles;

- Level 1 (green) - Interference can be tolerated;

- Level 2 (yellow) - Interference could be conditionally allowed;

- Level 3 (red) - Interference is not allowed;

- Level 4 (black) - Interference is impossible.

A weight factor imp is attributed to each level, allowing the algorithm to accept routes that cross less important obstacles. The penalty term is then defined by the following expression (again replacing a linear by an exponential function):

$$
\text { penal }_{\text {obst }}=\exp \left(\sum_{i=1}^{\text {nInter }} i m p\right)-1
$$




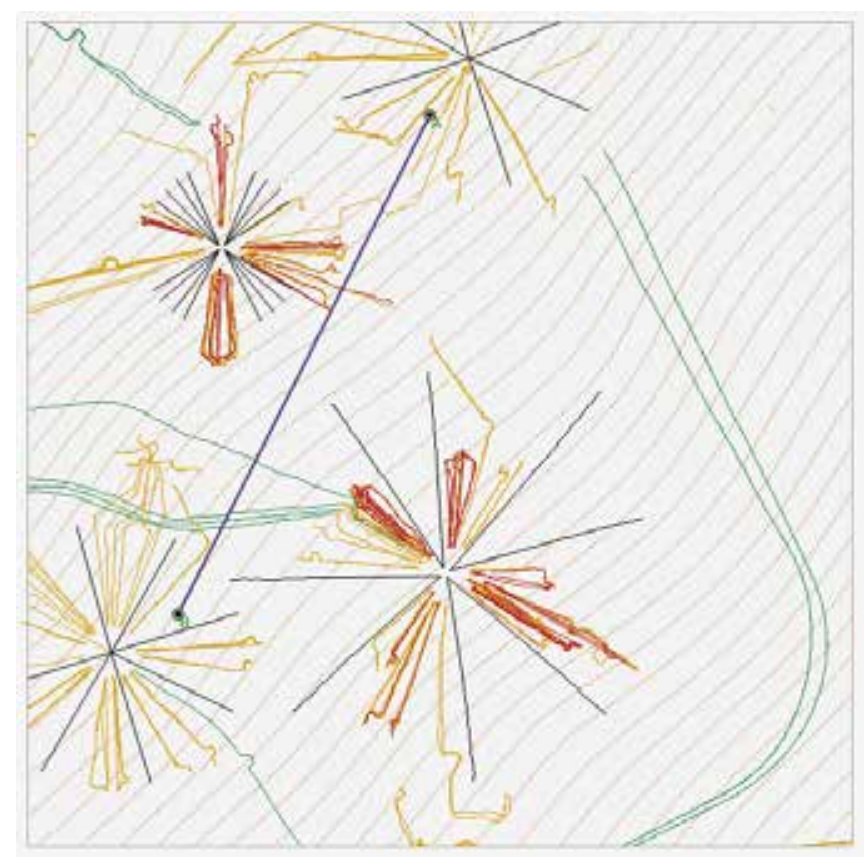

Figure 4. Representation of obstacles.

\subsubsection{Minimum Length of Straight Sections}

The pipeline launching operation may require straight sections between two consecutive curves, to allow proper space for the maneuvering of the launching ship. Straight sections are already incorporated in the route parameterization described before, but it is desirable that a minimum length $L_{\min }$ be respected. This is accomplished by the following penalty term that is introduced whenever the route presents a straight section with length $L_{\text {measure }}$ lower than $L_{\text {min }}$ :

$$
\text { penal }_{L \min S t r}= \begin{cases}\frac{L_{\min }-L_{\text {measure }}}{L_{\min }} & \text { if } L_{\text {measure }}<L_{\min } \\ 0 & \text { if } L_{\text {measure }} \geq L_{\min }\end{cases}
$$

Also to comply with installation requirements, the route should begin and end with a straight section. Thus, to encourage route configurations with this characteristic, the following penalty term is introduced whenever the length of the initial or final straight sections ( $\left.L_{\text {measure }}\right)$ is less than a given minimum value $\left(L_{m i n}\right)$ :

$$
\text { penal }_{L \text { min BeginEnd }}= \begin{cases}\frac{L_{\min }-L_{\text {measure }}}{L_{\min }} & \text { if } L_{\text {measure }}<L_{\text {min }} \\ 0 & \text { if } L_{\text {measure }} \geq L_{\min }\end{cases}
$$

\subsubsection{Minimum Radius of Curvature}

During the pipeline launching process, curves with small radius can lead the pipe to slide sideways, leaving the predefined route. To avoid such situations and encourage routes with radius of curvature larger than a given value $R_{\text {routemin }}$ (function of the pipe-soil friction coefficient $\mu$, pipe weight $w_{s}$ and residual pipe tension $T_{\text {residual }}$ at its equilibrium configuration after installation), the following penalty term is introduced: 


$$
\begin{aligned}
& R_{\text {routemin }}=\frac{T_{\text {residual }}}{\mu^{*} w_{s}} \\
& \text { penal }_{R \min }= \begin{cases}\sum_{i=1}^{n N o d e s P e n a l} \frac{\left(R_{\text {routemin }}-R_{i}\right)}{R_{\text {routemin }}} & \text { if } R_{i}<R_{\text {routemin }} \\
0 & \text { if } R_{i} \geq R_{\text {routemin }}\end{cases}
\end{aligned}
$$

\subsubsection{Longitudinal Declivity}

Another important geographical-topographical aspect, related to the seabottom bathymetry, is the slope on which the pipeline is set. Values for the declivity of each point of the route can be calculated from the isobathymetric lines and interpolating at the seabottom grid surface. Whenever the declivity of the pipe $\left(\theta_{L}\right)$ exceeds a given limit $\left(\theta_{L_{-} L i m}\right)$, the following penalty term can then be introduced, averaged by the number of points on the route $n N o d e s:$

$$
\text { penal }_{\text {Decliv }}=\frac{\sum_{i=1}^{n \text { NodesPenal }} \exp \left(\theta_{L}^{i}-\theta_{L_{-} \text {Lim }}\right)}{n \text { Nodes }} \text { if } \theta_{L}^{i}>\theta_{L_{-} \text {Lim }}
$$

\subsubsection{Stability Criteria}

As mentioned before, the stability criteria are the main scope of this work. Roughly speaking, for vertical stability these criteria are associated with the ratio between the lift/buoyancy force and the pipe weight; for lateral stability, to the ratio between soil resistance and hydrodynamic forces.

The penalty terms associated with the vertical and lateral stability are defined by the following expressions:

$$
\begin{aligned}
& \text { penal }_{\text {Vertstab }}=\frac{\sum_{i=1}^{n \text { Nodespenal }} S F-\gamma_{s c_{-2}}^{i}}{n \text { Nodes }} \text { if } \gamma_{s c_{-2}}^{i}<S F \\
& \text { penal }_{\text {Latstab }}=\frac{\sum_{i=1}^{n N o d e s P e n a l} S F-\gamma_{s c_{-} y}^{i}}{n N o d e s} \text { se } \gamma_{\text {sc } \_y}^{i}<S F
\end{aligned}
$$

The next section will describe the calculation of the factors $\gamma_{s c_{-} y}$ and $\gamma_{s_{-} z}$ involved in these expressions, based on the safety factors $(\gamma)$ recommended by DNV-RP-F109 [6]. Note that, in this implementation, the route only violates the constraint when these calculated factors are less than a safety factor $(S F)$. Regarding lateral stability, in section 3.6.3 of [6] different safety factor values are proposed for the North Sea and Gulf of Mexico. In the current implementation of the tool, one can use the default value of 1.1 or enter user-defined values.

\section{ON-BOTTOM STABILITY CRITERIA}

The DNV-RP-F109 code [6] describes three different methodologies for analysis and verification of the lateral stability of pipelines:

i. Absolute Lateral Static Stability”: Ensuring “absolute static stability” with zero 
displacement, that is, ensuring that the hydrodynamic loads acting on the pipe are less than the soil resistance;

ii. "Generalized Lateral Stability Method": Ensuring "no break-out”, for a "virtually stable” pipe, allowing small displacements (less than about one half diameter), taking advantage of the passive resistance of the soil and ensuring that the pipe does not move out of its cavity, with maximum displacements independent of time;

iii. "Dynamic Lateral Stability Analysis": Allowing “accumulated displacements", with the pipe able to break out of (and return to) its cavity, and the soil resistance is dependent on the time-history of pipe displacements.

These approaches proposed in [6] may be associated with analytical expressions, precalibrated curves or dynamic FE analyses. In the case of the Absolute Lateral Static Stability method, static analytical expressions provides safety factors associated with the ratio between hydrodynamic loads and horizontal soil resistance; in the case of the Generalized Lateral Stability method, pre-calibrated curves provide the minimum required weight for a given maximum allowable displacement.

The item below describes the methodology of Absolute Static Stability which will be considered in this work. The work [5] presents the first two methodologies (Absolute Stability and Generalized Stability) and compares the results presented by the two methodologies.

\subsection{Absolute Lateral Static Stability}

The Absolute Stability Method is the most simple project methodology and is based on the equilibrium of forces (Fig. 5). In addition some premises are adopted:

- Pipe displacements are not tolerated;

- The horizontal resultant of the hydrodynamic loads acting on the pipe should be lower than the soil resistance, and the lift should be lower than the submerged pipe weight.

\section{LIFT FORCE}

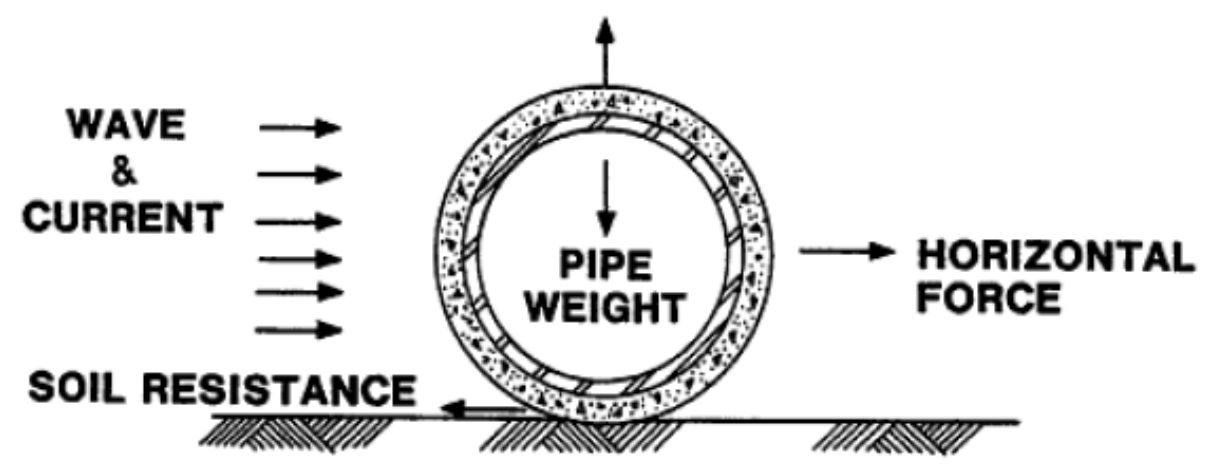

Figure 5. Forces acting on the pipe.

Besides that, some remarks are taken into account for the stability calculation:

i. The wave load is accounted as a single component of a unidirectional regular wave;

ii. Speeds and accelerations are measured at pipe level using the linear wave theory;

iii. Hydrodynamic loads are calculated by means of the DNV RP-F109 [6] recommended formulation, a correlative to the Morison formulation; 
iv. Soil resistance has two parts, one of friction force, dependent on the static friction coefficient $\mu$ and the normal force acting on the soil, and another corresponding to the FR passive resistance from the pipe's initial penetration on the soil.

Therefore, according to DNV RP-F109 [6], for a pipe to be considered stable two conditions must be met, establishing safety factors $\gamma_{s c}$ that ensure lateral and vertical stability.

It's noteworthy that the $\gamma_{s c}$ safety factors, originally derived from DNV RP-F109 [6], consider leveled floors. Meanwhile, for real bathymetry, it is necessary to consider the declivity of the bathymetric floor.

As a result the criteria was extended to consider slopes, in order to assure that the optimization process favors relatively leveled trajectories (perpendicular to the pipe), avoiding sliding on regions where bathymetry shows increased transversal slopes (Fig. 6).

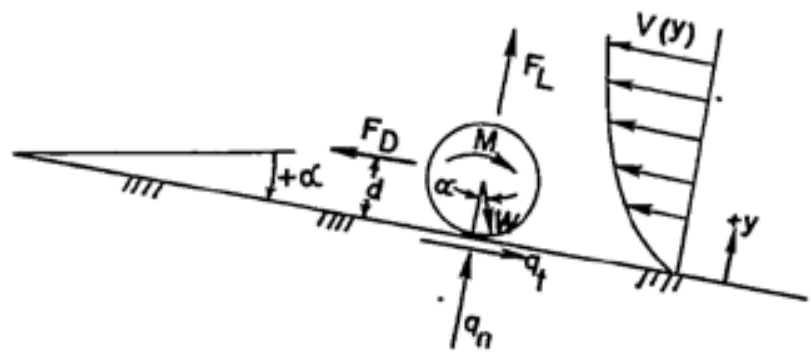

\section{(a) UP SLOPE VELOCITY}

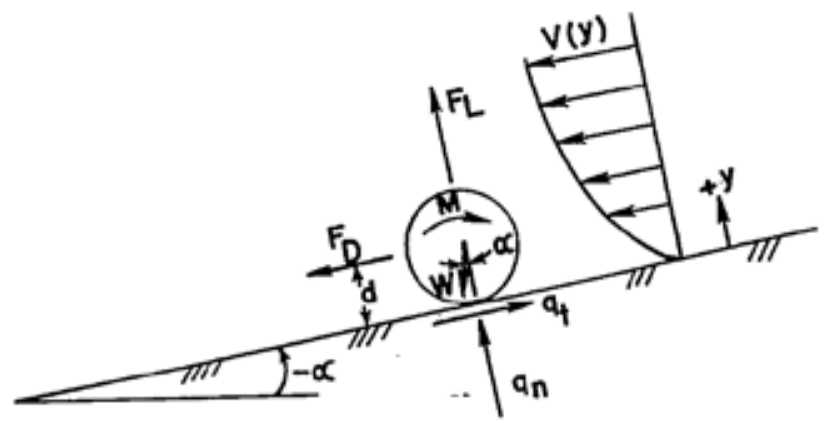

(b) DOWN SLOPE VELOCITY

Figure 6. Hydrodynamic Forces considering bathymetry slopes.

The first condition corresponds to the static lateral equilibrium, where the equilibrium between the horizontal components of the hydrodynamic forces and the soil resistance is checked:

$$
\gamma_{s c_{-} y}=\frac{\mu \cdot w_{s} \cdot \cos \left(\theta_{t}\right)+F_{R}}{\left|\left(F_{D}+F_{I}\right)+w_{s} \cdot \sin \left(\theta_{t}\right)+\mu \cdot F_{L}\right|}
$$

where $\gamma_{s c_{-} y}$ is the lateral safety factor; $F_{D}$ is the drag force; $F_{I}$ is the inertia force; $F_{L}$ is the lift force; $w_{s}$ is the pipe's submerged weight; $\mu$ is the coefficient of friction; e $F_{R}$ is the soil's passive resistance force; $\theta_{t}$ is the transversal inclination angle.

The second condition corresponds to the static vertical equilibrium, where the submerged pipe weight $w_{s}$ and the lift force $F_{L}$ loads are checked: 


$$
\gamma_{s C_{-} z}=\frac{w_{s} \cdot \cos \left(\theta_{t}\right)}{F_{L}}
$$

where $\gamma_{s c_{z} z}$ is the vertical safety factor.

\subsection{Consideration of the Required Ballast Weight}

The following procedure (Fig. 7) was designed to insert the $W_{\text {req }}$ required ballast weight factor into the objective function formulation (item 3.2.2):

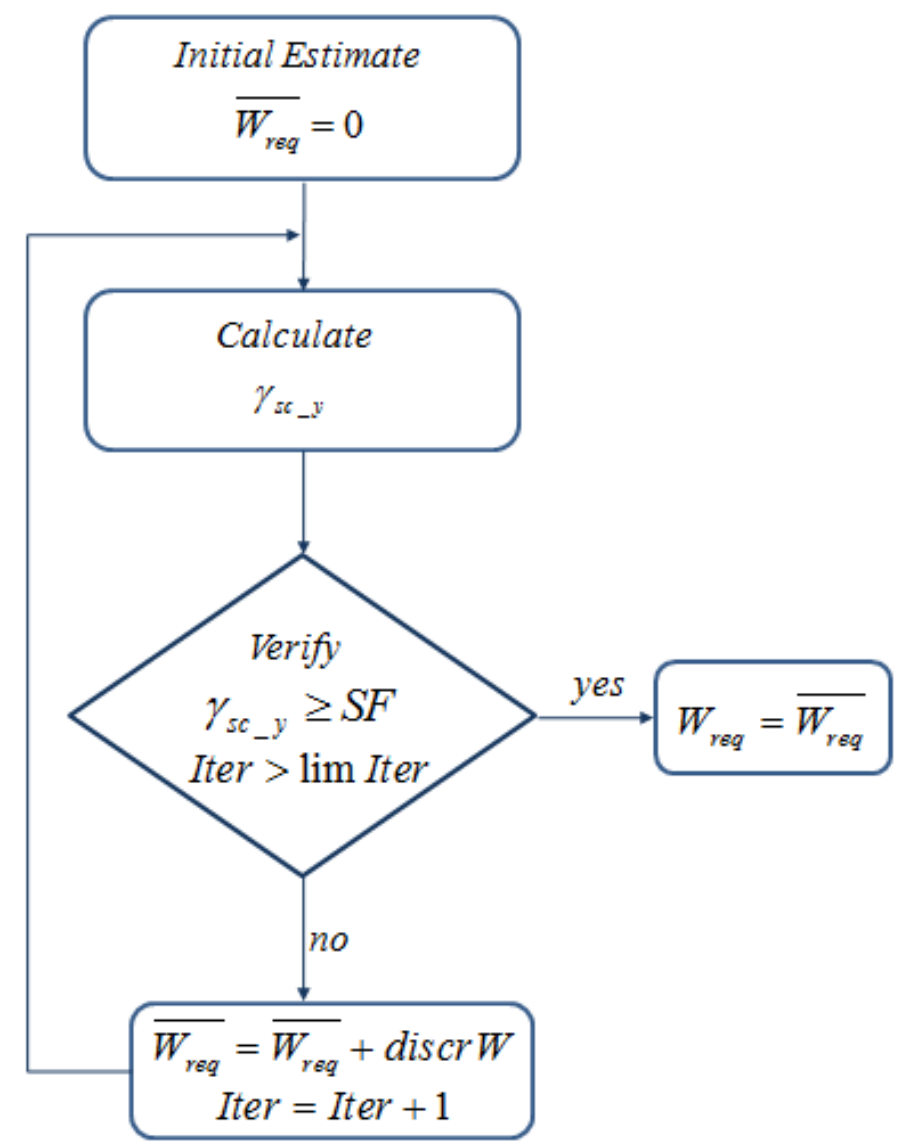

Figure 7. Required ballast weight calculation flowchart.

This procedure aims to gradually increase the pipe's ballast weight $\left(W_{\text {req }}\right)$ until it provides a safety factor $\left(\gamma_{s c_{-} y}\right)$ that meets the defined minimum safety factor $(S F)$, which has the value of 1.1 for this work. Therefore, using $W_{\text {req, }}$, the pipe's submerged weight $\left(w_{s}\right)$ is calculated and, subsequently, the hydrodynamic forces and soil resistance calculation is applied to define $\gamma_{s c_{-} y}$ for the absolute criteria.

Additionally, it is noteworthy that the calculated ballast weight $\left(W_{\text {req }}\right)$ does not consider a concrete thickness on the pipe itself, complying only with burying and concrete mattress ballast techniques. 


\section{CASE STUDIES}

The objective of the case studies presented here is to assess the influence of the stability criteria on the definition of the optimal pipeline route.

\subsection{Scenario}

For this case study the hypothetical scenery shown in Fig. 8 was adopted. The deepest point $\mathrm{A}$ is located at around $640 \mathrm{~m}$ deep, whereas the shallowest point $\mathrm{B}$ is located at around 120m deep. Soil and pipe properties are presented at Table 1 and 2, respectively.

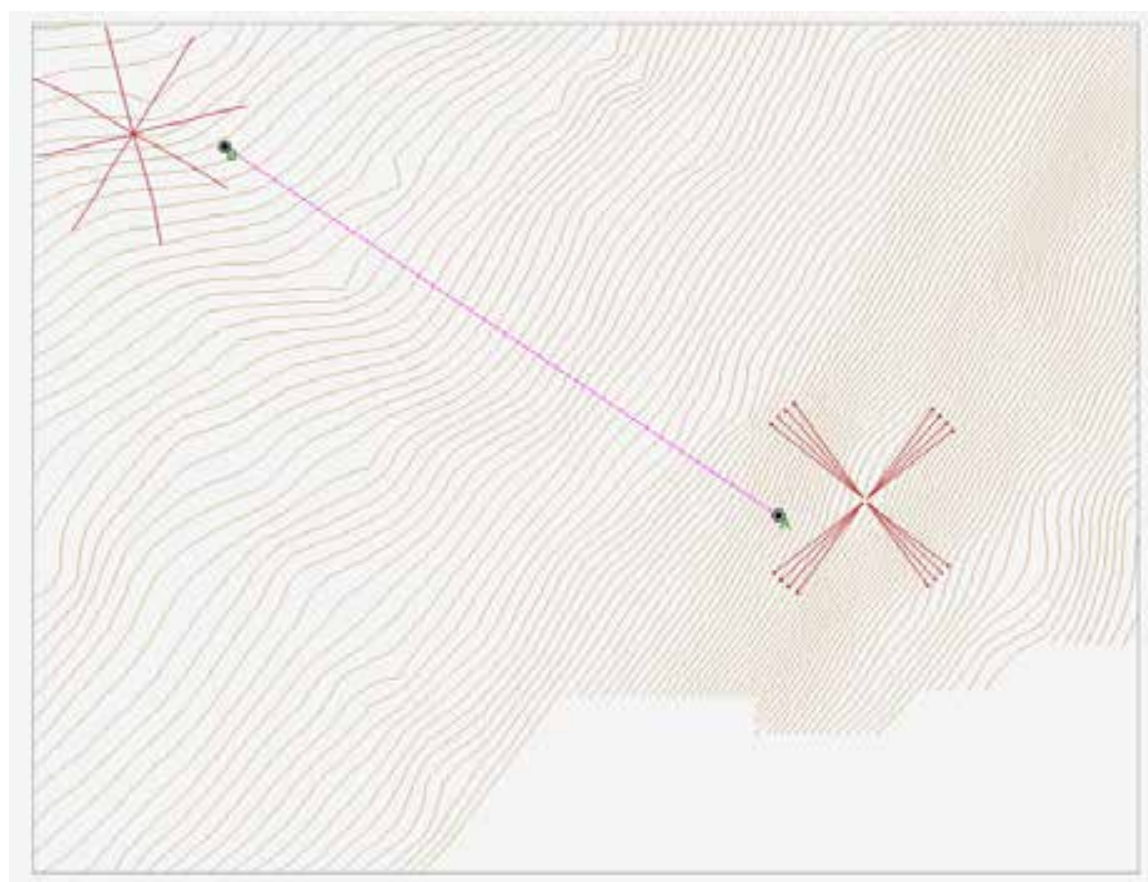

Figure 8. Bathymetry.

Table 1. Soil Properties.

\begin{tabular}{cc}
\hline \multicolumn{2}{c}{ Properties } \\
\hline Type Soil & Sand \\
\hline$\mu$ (Friction Coefficient) & 0.7 \\
\hline$\gamma s^{\prime}$ (Submergd unit soil weight) & $13500 \mathrm{~N} / \mathrm{m}^{3}$ \\
\hline
\end{tabular}


Table 2. Pipeline Properties.

\begin{tabular}{cc}
\hline \multicolumn{2}{c}{ Properties } \\
\hline Outside Diameter OD & $0.32385 \mathrm{~m} / 123 / 4 \mathrm{in}$ \\
\hline Wall thickness $\mathrm{t}_{\mathrm{s}}$ & $0.01905 \mathrm{~m} / 3 / 4 \mathrm{in}$ \\
\hline Steel specific weight $\rho_{\mathrm{s}}$ & $77000 \mathrm{~N} / \mathrm{m}^{3}$ \\
\hline Corrosion $\quad$ Thickness $\mathrm{t}_{\mathrm{p}}$ & $0.076 \mathrm{~m} / 3$ in \\
Coating Spec. Weight $\rho_{\mathrm{p}}$ & $8826 \mathrm{~N} / \mathrm{m}^{3}$ \\
\hline
\end{tabular}

Each candidate route of the optimization process has its on-bottom stability assessed under a set of loading cases, comprised by a combination of current and waves presented in the following tables. Table 3 presents the near-bottom current velocities for eight directions (N, NE, E, SE, S, SW, W and NW) and two return periods (10 and 100 years). Table 4 presents the wave data for the shallower range (recalling that wave loadings are not important in deep waters).

The steady near bottom current velocities perpendicular to the pipeline is calculated by applying a factor to take into account the effect of the bottom boundary layer and the directionality. The near bottom water velocities due to waves are calculated using the JONSWAP spectral model.

Table 3. Near bottom extreme current velocity $(\mathrm{m} / \mathrm{s})$.

\begin{tabular}{ccc}
\hline & \multicolumn{2}{c}{ Return Period (years) } \\
\hline Direction & $\mathbf{1 0}$ & $\mathbf{1 0 0}$ \\
\hline N & 0.54 & 0.65 \\
NE & 0.75 & 0.96 \\
E & 0.62 & 0.74 \\
SE & 0.65 & 0.77 \\
S & 0.96 & 1.18 \\
SW & 1.09 & 1.41 \\
W & 0.63 & 0.75 \\
NW & 0.55 & 0.61
\end{tabular}


Table 4. Extreme wave parameters.

\begin{tabular}{|c|c|c|c|}
\hline \multirow[b]{2}{*}{ Direction } & \multirow[b]{2}{*}{ Parameter } & \multicolumn{2}{|c|}{$\begin{array}{c}\text { Return Period } \\
\text { (years) }\end{array}$} \\
\hline & & 10 & 100 \\
\hline \multirow[t]{2}{*}{$\mathrm{N}$} & $\mathrm{H}_{\mathrm{s}}$ & 4.74 & 5.01 \\
\hline & $\mathrm{T}_{\mathrm{P}}$ & 9.20 & 9.56 \\
\hline \multirow[t]{2}{*}{ NE } & $\mathrm{H}_{\mathrm{s}}$ & 4.88 & 5.17 \\
\hline & $\mathrm{T}_{\mathrm{P}}$ & 9.47 & 9.77 \\
\hline \multirow[t]{2}{*}{$\mathrm{E}$} & $\mathrm{H}_{\mathrm{s}}$ & 4.34 & 4.87 \\
\hline & $\mathrm{T}_{\mathrm{P}}$ & 9.90 & 10.40 \\
\hline \multirow[t]{2}{*}{$\mathrm{SE}$} & $\mathrm{H}_{\mathrm{s}}$ & 5.72 & 6.53 \\
\hline & $\mathrm{T}_{\mathrm{P}}$ & 10.28 & 11.63 \\
\hline \multirow[t]{2}{*}{$\mathrm{S}$} & $\mathrm{H}_{\mathrm{s}}$ & 6.19 & 7.10 \\
\hline & $\mathrm{T}_{\mathrm{P}}$ & 13.54 & 14.35 \\
\hline \multirow[t]{2}{*}{ SW } & $\mathrm{H}_{\mathrm{s}}$ & 7.16 & 7.84 \\
\hline & $\mathrm{T}_{\mathrm{P}}$ & 14.78 & 15.55 \\
\hline \multirow[t]{2}{*}{ W - NW } & $\mathrm{H}_{\mathrm{s}}$ & 3.57 & 3.88 \\
\hline & $\mathrm{T}_{\mathrm{P}}$ & 8.22 & 8.51 \\
\hline
\end{tabular}

\subsection{Results}

\subsubsection{Optimization with Stability as Penalty}

In order to evaluate the influence of hydrodynamic stability in the route optimization, optimizations were performed by activating and deactivating the stability criteria, considering the stability as a constraint of the problem (items 3.2.1 and 3.3.6).

The "optimal" routes for the scenario are illustrated in Fig. 9. Table 5 indicates the length of the routes.

Table 5. Optimization result using stability as penalty.

\begin{tabular}{cc}
\hline Stability Criteria & Route Lengths \\
\hline Without & $12,530 \mathrm{~m}$ \\
\hline Absolute & $14,943 \mathrm{~m}$
\end{tabular}




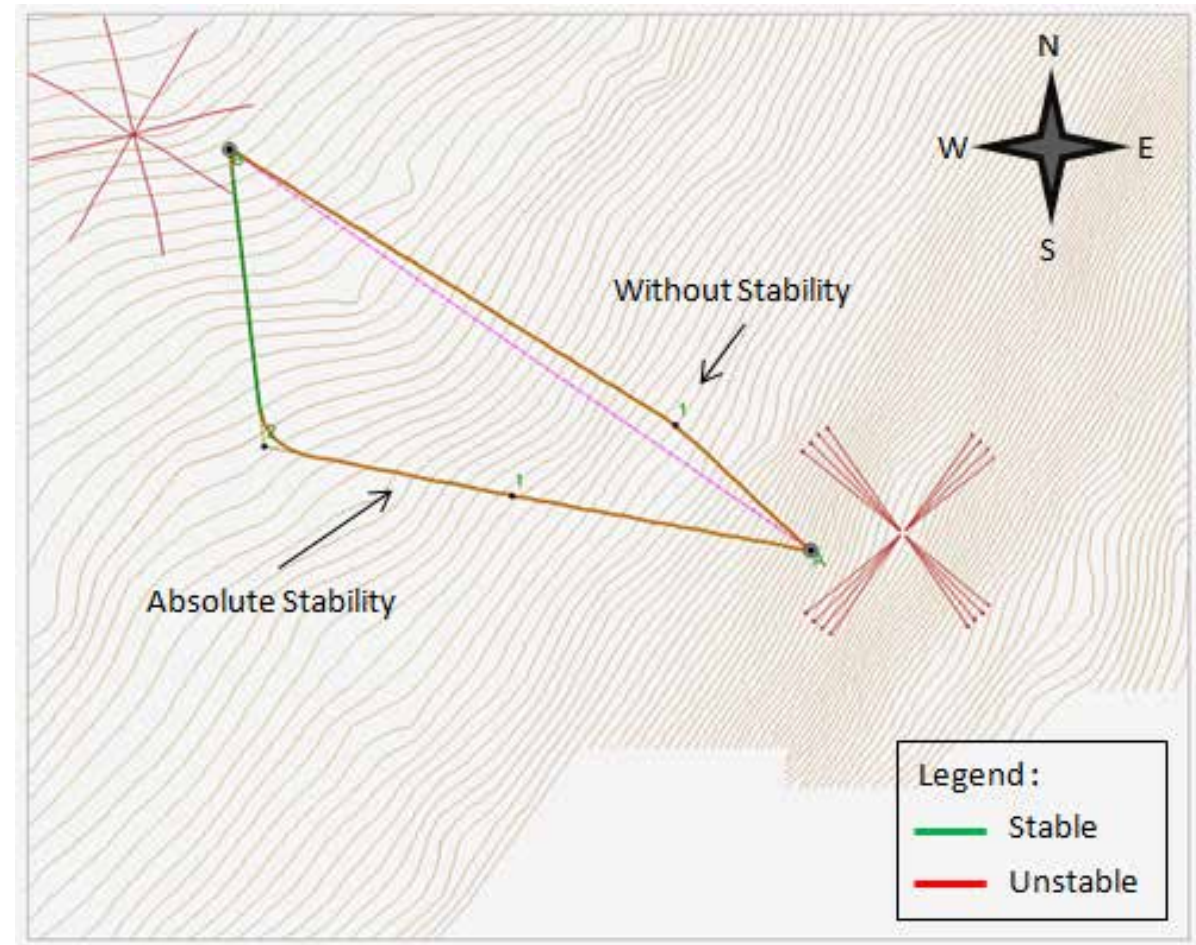

Figure 9. Optimization result using stability as penalty.

Observing the results Fig. 9, the route generated by the optimization run without the stability criteria is very close to the straight line between the endpoints, just enough to avoid the penalty associated with the longitudinal declivity (recalling that in these runs the limit value is set to $5^{\circ}$ ). However, this route is highly unstable, since it is almost perpendicular to the most severe directions of the environmental loadings (the SW and S directions, as can be observed in Table 3).

On the other hand, the routes generated with the stability criteria are longer but have more segments stable. This is because the optimization algorithm tends to generate routes roughly aligned with the NE-SW or N-S directions, in order to reduce the resultant environmental loads normal to the pipeline axis, particularly in the shallowest section where the environmental loads are most significant.

\subsubsection{Optimization with Stability as Ballast Weight}

To evaluate the minimum weight required to stabilize a route has been inserted a discretizing 100 to $100 \mathrm{~N} / \mathrm{m}$ for the ballast weight. Additionally, stabilization of the route is only reached when the weight of the element results in a safety factor greater than 1.1.

Thus, to evaluate the influence of the required ballast weight on the route (item 3.2.2 and 0 ), optimizations were performed by varying the relative weighting to the length of the pipe ( $\left.k_{\text {pipe }}\right)$ and the relative weighting to the ballast $\left(k_{\text {ballast }}\right)$. Therefore, the higher the $k_{\text {pipe }}$, the lower the route, but the higher the required ballast weight, and vice-versa.

The results are compared in terms of fitness found, of the length, of the ballast weight and of the geometry of the optimal route obtained. Moreover, the figures have color map, in which the reddish gives higher weight to the route, the green gives lower weight and the 
yellow gives an intermediate weight.

The "optimal" routes for the scenario are illustrated in Fig. 10. Table 6 indicates the length of the routes.

Table 6. Optimization result using stability as Ballast weight.

\begin{tabular}{cccc}
\hline Stability Criteria & Route Lengths & Total Weight & Average Weight \\
\hline $\begin{array}{c}k_{\text {pipe }}=1 \\
k_{\text {ballast }}=0.01\end{array}$ & $14,588 \mathrm{~m}$ & $1,328 \mathrm{KN}$ & $91 \mathrm{~N} / \mathrm{m}$ \\
\hline $\begin{array}{c}k_{\text {pipe }}=10 \\
k_{\text {ballast }}=0.01\end{array}$ & $13,461 \mathrm{~m}$ & $2,127 \mathrm{KN}$ & $158 \mathrm{~N} / \mathrm{m}$ \\
\hline $\begin{array}{c}k_{\text {pipe }} \\
=\end{array}=50$ & $12,619 \mathrm{~m}$ & $3,054 \mathrm{KN}$ & $242 \mathrm{~N} / \mathrm{m}$
\end{tabular}

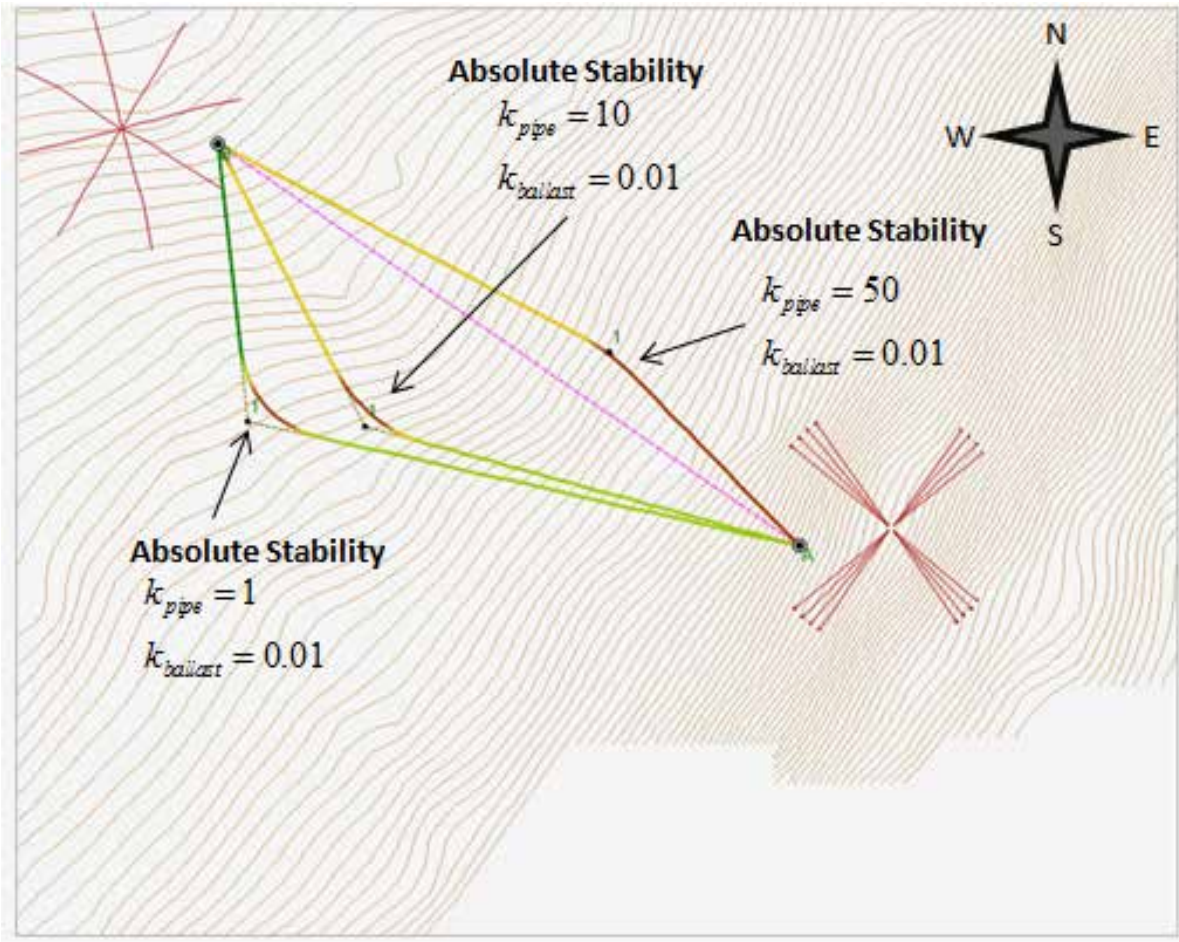

Figure 10. Optimization result using stability as Ballast weight.

The result shown in Fig. 10 presents that as the $k_{\text {pipe }}$ increases as the route tends to reduce the length, however the total ballast weight increases.

Analyzing the route with $k_{\text {pipe }}=1$ can be observed that the shallower section has ballast weight 0 (section with more intense green color) that because the algorithm tends to align this section with prevailing loads of NE-SW and N-S. As in these depths environmental loading is more significant if the pipe receives the direct action, the weights associated with ballast would be very high. However, in the deepest part the leading load acts almost directly on the pipeline, requiring a small weight $(100 \mathrm{~N} / \mathrm{m})$ for the route is stable.

In the route with $k_{\text {pipe }}=50$, which governs is the length of the route, in this way the weight required is higher. The route with $k_{\text {pipe }}=10$ shows a configuration intermediate simultaneously trying to reduce the length of the route and the ballast weight is necessary to stabilize it. 


\section{FINAL REMARKS}

Based on the study presented, we can see that the calculation of stability influences considerably the process of finding the best route, showing a new concept for tracing the route of submarine pipelines in relation to the verification of hydrodynamic stability concurrently with the project route.

Additionally, the results confirm the need for optimizing the ballast weight of the pipe together with the length of the route in order to avoid excessively long configurations to achieve stability and avoid excessive ballast.

Therefore, the optimization tool developed provides consistent results, satisfactory and with reduced computational time, which corroborates the feasibility of the coupling of hydrodynamic stability with the geometric design of pipeline route.

\section{Acknowledgements}

The authors would like to acknowledge the support of the Research \& Development Center of PETROBRAS (CENPES).

\section{REFERENCES}

[1] Vieira LT, de Lima BSLP, Evsukoff AG, Jacob BP (2003), Application of genetic algorithms to the synthesis of riser configurations. In: Proceedings of the 22th International Conference on Offshore Mechanics and Arctic Engineering, CD-ROM, paper OMAE2003-37231, Cancun, Mexico.

[2] de Lima BSLP, Jacob BP, Ebecken NFF. A hybrid fuzzy/genetic algorithm for the design of offshore oil production risers. Int J Numer Meth Eng 64:1459-1482, DOI: 10.1002/nme.1416, 2005.

[3] Pina AA, Albrecht CH, de Lima BSLP, Jacob BP. Tailoring the particle swarm optimization algorithm for the design of offshore oil production risers. Optimization and Engineering, OnlineFirst, DOI 10.1007/s11081-009-9103-5, 2010.

[4] Vieira IM, Albrecht CH, de Lima BSLP, Jacob BP, Rocha DM, Cardoso CO. Towards a Computational Tool for the Synthesis and Optimization of Submarine Pipeline Routes. Procs. of the Twentieth International Offshore and Polar Engineering Conference ISOPE 2010, Beijing, China.

[5] Lima Jr. MHA, Baioco JS, Albrecht CH, de Lima BSLP, Jacob BP, Rocha DM, Cardoso CO, 2011. Synthesis and Optimization of Submarine Pipeline Routes Considering OnBottom Stability Criteria. In: Proceedings of the ASME 2011 30th International Conference on Ocean, Offshore and Arctic Engineering, OMAE 2011, Rotterdam, Holand.

[6] Recommended Practice DNV-RP-F109, On Bottom Stability Design of Submarine Pipelines, Det Norske Veritas, 2008.

[7] Baioco JS, Lima Jr. MHA, Albrecht CH, de Lima BSLP, Jacob BP, Rocha DM, Cardoso CO, 2011. Offshore Pipeline Routes Evaluating Weight Ballast to Achieve On-Bottom Stability. In: Proceedings of the Iberian Latin American Congress on Computational 
Methods in Engineering, CILAMCE 2011, Ouro Preto, Brazil.

[8] Goldberg DE. Genetic Algorithms in Search, Optimization and Machine Learning. Addison-Wesley, 1989. 